\title{
Observations of the GRB Afterglow ATLAS17aeu and Its Possible Association with GW 170104
}

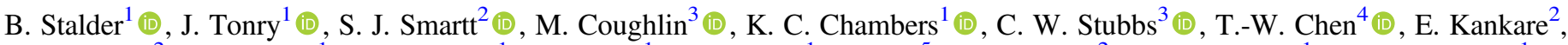
K. W. Smith ${ }^{2}$, L. Denneau ${ }^{1}$, A. Sherstyuk ${ }^{1}$, A. Heinze ${ }^{1}$, H. Weiland ${ }^{1}$, A. Rest ${ }^{5}$, D. R. Young ${ }^{2}$ (D) M. E. Huber ${ }^{1}$ (1) , H. Flewelling ${ }^{1}$ (i), T. Lowe ${ }^{1}$, E. A. Magnier ${ }^{1}$ (1), A. S. B. Schultz ${ }^{1}$, C. Waters ${ }^{1}$ (D), R. Wainscoat ${ }^{1}$ (D) M. Willman ${ }^{1}$, D. E. Wright ${ }^{6}$, J. Chu ${ }^{1}$,

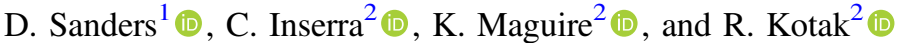

${ }^{1}$ Institute for Astronomy, University of Hawaii, 2680 Woodlawn Drive, Honolulu, HI 96822, USA; stalder@ifa.hawaii.edu

${ }^{2}$ Astrophysics Research Centre, School of Mathematics and Physics, Queens University Belfast, Belfast BT7 1NN, UK

${ }^{3}$ Department of Physics, Harvard University, Cambridge, MA 02138, USA

${ }^{4}$ Max-Planck-Institut für Extraterrestrische Physik, Giessenbachstraße 1, D-85748, Garching, Germany

5 Space Telescope Science Institute, 3700 San Martin Drive, Baltimore, MD 21218, USA

${ }^{6}$ School of Physics and Astronomy, University of Minnesota, 116 Church Street SE, Minneapolis, MN 55455-0149, USA

Received 2017 May 31; revised 2017 September 6; accepted 2017 October 22; published 2017 November 28

\begin{abstract}
We report the discovery and multiwavelength data analysis of the peculiar optical transient, ATLAS17aeu. This transient was identified in the sky map of the LIGO gravitational wave event GW 170104 by our ATLAS and PanSTARRS coverage. ATLAS17aeu was discovered $23.1 \mathrm{hr}$ after GW 170104 and rapidly faded over the next three nights, with a spectrum revealing a blue featureless continuum. The transient was also detected as a fading X-ray source by Swift and in the radio at 6 and $15 \mathrm{GHz}$. The gamma-ray burst GRB 170105A was detected by three satellites $19.04 \mathrm{hr}$ after GW 170104 and $4.10 \mathrm{hr}$ before our first optical detection. We analyze the multiwavelength fluxes in the context of the known GRB population and discuss the observed sky rates of GRBs and their afterglows. We find it statistically likely that ATLAS17aeu is an afterglow associated with GRB 170105A, with a chance coincidence ruled out at the $99 \%$ confidence or $2.6 \sigma$. A long, soft GRB within a redshift range of $1 \lesssim z \lesssim 2.9$ would be consistent with all the observed multiwavelength data. The Poisson probability of a chance occurrence of GW 170104 and ATLAS17aeu is $p=0.04$. This is the probability of a chance coincidence in 2D sky location and in time. These observations indicate that ATLAS17aeu is plausibly a normal GRB afterglow at significantly higher redshift than the distance constraint for GW 170104 and therefore a chance coincidence. However, if a redshift of the faint host were to place it within the GW 170104 distance range, then physical association with GW 170104 should be considered.
\end{abstract}

Key words: gamma-ray burst: general - gamma-ray burst: individual (GRB 170105) - gravitational waves - stars: black holes

\section{Introduction}

The Advanced LIGO experiment began detecting gravitational waves from the merging of black hole $(\mathrm{BH})$ binary systems in 2015 (Abbott et al. 2016b). In addition to being the first direct detection of gravitational waves, these first three detections showed that remarkably high stellar mass BHs exist, with the highest mass system (GW 150914) resulting from the coalescence of a $36 M_{\odot}$ and $29 M_{\odot}$ system. As of yet, no probable electromagnetic counterparts to these events have been found. However, the Fermi Gamma-ray Burst Monitor (GBM) detected a weak, hard X-ray transient temporally coincident with GW 150914 (Connaughton et al. 2016), but this has not been confirmed to be physically associated, and its astrophysical nature has been disputed (Greiner et al. 2016).

The second science run of the Advanced LIGO experiment (designated O2) started MJD $=57722$ (2016 November 30) with a short holiday break between MJD $=57744$ and MJD $=57756$. Shortly after the break, the internal system distributed the alert of a candidate gravitational wave (GW) transient, designated as event G268556 at 2017 January 04 10:11:58.599 UTC or MJD $=57757.42498378$. It was later given the name GW 170104 after the offline analysis provided very strong confirmation of its astrophysical origin (as presented in Abbott et al. 2017). The $90 \%$ probability area of the associated GW sky map is 2000 square degrees or $5 \%$ of the sky. Throughout this paper, we use the LALInference sky map released on 2017 May 02 by LIGO, i.e., LALInference_f.fits (Veitch et al. 2015). The estimated binary BH masses are $31.2_{-6.0}^{+8.4} \quad M_{\odot}$ and $19.4_{-5.9}^{+5.3} \quad M_{\odot}$ at a luminosity distance of $880_{-390}^{+450} \mathrm{Mpc}$, corresponding to a redshift of $0.18_{-0.07}^{+0.08}$ (Abbott et al. 2017).

As with previous LIGO events, many teams with electromagnetic follow-up facilities observed the corresponding sky map of this event (e.g., for a summary of the GW 150914 effort see Abbott et al. 2016a). Here we introduce the Asteroid Terrestrial-impact Last Alert System (ATLAS; Tonry 2011), a full-time near-Earth asteroid survey, and describe its early observations of GW 170104 in combination with our established Pan-STARRS follow-up program (Chambers et al. 2016; Smartt et al. 2016a, 2016b) During the course of our coordinated ATLAS and Pan-STARRS observations, we identified a bright optical transient, ATLAS17aeu, discovered $23.1 \mathrm{hr}$ after GW 170104 and observed to be rapidly fading over $2 \mathrm{hr}$. We discuss the nature of this optical transient and its relation to both the gravitational wave source GW 170104 and the gamma-ray burst GRB 170105A detected by several gamma-ray satellite missions. Throughout this paper, we use the same cosmological parameters as adopted by LIGO, $H_{0}=69 \mathrm{~km} \mathrm{~s}^{-1}, \Omega_{\mathrm{M}}=0.31, \Omega_{\Lambda}=0.69$. 


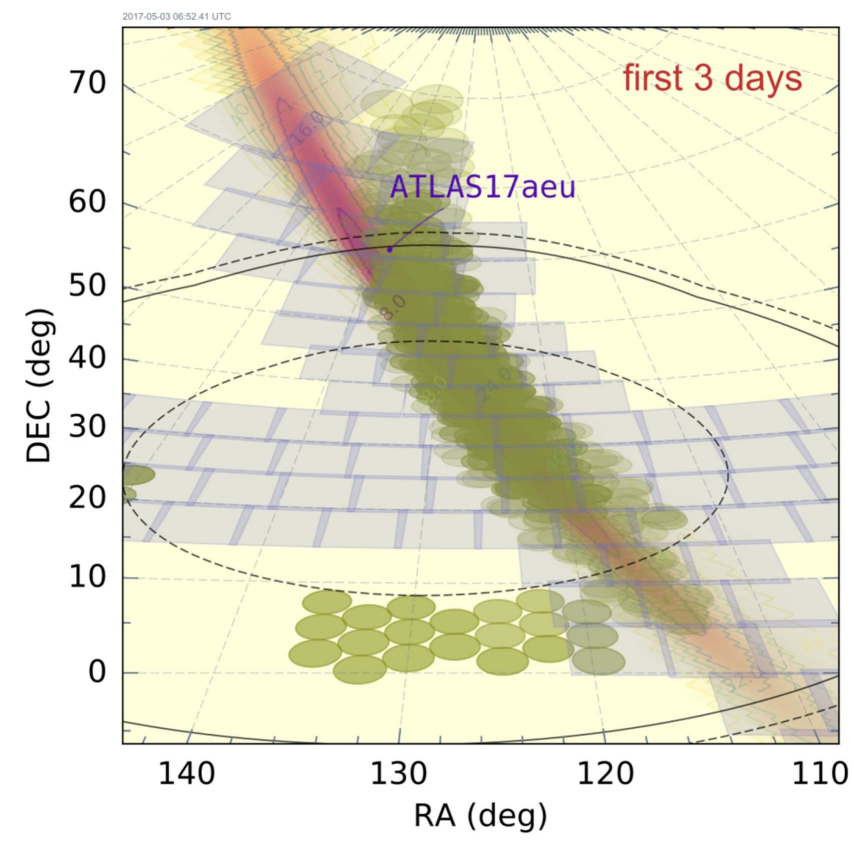

Figure 1. LALInference likelihood map (LALInference_f.fits) showing ATLAS (purple squares) and Pan-STARRS1 pointings (green circles). The black solid circle is the best-estimate localization of GRB 170105A, and the black dashed circles represent the Konus-Integral triangulation annulus of Svinkin et al. (2017).

\section{Discovery of ATLAS17aeu and Follow-up Optical Observations}

The ATLAS system ${ }^{7}$ currently consists of two $0.5 \mathrm{~m} \mathrm{f} / 2$ wide-field telescopes (Tonry 2011), of which only the Haleakala unit was in operation at the time of these observations. The ATLAS sensor is a single thermoelectrically cooled STA1600 detector with 1.86 arcsec per pixel platescale $(10.56 \mathrm{k} \times 10.56 \mathrm{k}$ pixels $)$, giving a 29.2 square degree field of view. As stated above, GW 170104 was detected on $\mathrm{MJD}=57757.42498378$ (Abbott et al. 2017) and the notification was sent to partners at 57757.70134 (6.6 hr later). Before the alert was released, and coincident with the GW detection time, ATLAS was observing a declination strip between $14^{\circ}<\delta<35^{\circ}$, cutting through the sky map (Figure 1) at this time, with the first pointing post-detection that encroached on the nonzero probability region taken at $\mathrm{MJD}=57757.44622(0.5 \mathrm{hr}$ after the GW detection $)$. One transient was found in the strip during this Hawaiian night of serendipitous survey operations (ATLAS17ace), but it is a known cataclysmic variable (CV) candidate with observed activity in CRTS and Pan-STARRS1 going back to 2014 (the object is CSS140914-075541+264619, PS1-14amh). When the LIGO GW event alert was processed and night subsequently fell in Hawaii, the ATLAS telescope began targeting the fields identified by the GW 170104 sky map (Figure 1), using the same cadence as normally employed for asteroid discovery and identification. These observations began at 57758.29696 or $20.9 \mathrm{hr}$ after the signal detection. The tiling started at the bottom of the banana-shaped sky map at R.A. = 108.79, decl. $=-7.662$ and continued north-eastward toward the top of the banana, finishing at $57758.46645(25.0 \mathrm{hr}$ after the signal detection) at sky position R.A. $=170.55$, decl. $=72.314$. Had

fallingstar.com there been no $6.6 \mathrm{hr}$ notification delay, ATLAS could have been observing within minutes of the LIGO detection as the event was well placed for Hawaiian night time observing. During this first night of dedicated observing, ATLAS covered about $42.6 \%$ of the enclosed probability, corresponding to a sky area of 1231 square degrees. The ATLAS system uses Canon 8-15 mm fisheye cameras that cover most of the visible sky from Haleakala and Mauna Loa. By coadding images immediately following the instant of GW 170104 and using an adjacent $m \sim 5.3$ star as a photometric reference, we can place a $3 \sigma$ limit for any optical transient at the location of ATLAS17aeu of $m>7.5$ for a 1-minute average brightness, $m>10$ for a 1-hour average, and $m>11$ between the instant of GW 170104 and the end of night in Hawaii.

We observed in a wide-band filter, designated "cyan" or " $c$," which roughly covers the SDSS/Pan-STARRS $g$ and $r$ filters, and maintained our cadence for identifying moving asteroids, which was to observe each footprint five times or more $(30 \mathrm{~s}$ exposures, slightly dithered) within about an hour of the first observation of each field. The automatic data-processing pipeline results in dark-subtracted, sky-flattened images as well as in difference images using a static-sky template generated from previous stacked data. Source extraction is accomplished on the normal and differenced images using DOPHOT (Schechter et al. 1993) and TPHOT (a custom-written package for pointspread function (PSF) fitting photometry on ATLAS images). Sources on the difference images are cataloged in a MySQL database and merged into objects if there are at least three detections from the five (or more) images. The objects are subject to a set of quality filters, a machine-learning algorithm, and human scanning, similar to those described for Pan-STARRS transient searches (Wright et al. 2015; Smartt et al. 2016b). ATLAS17aeu was discovered in the first image pass of this region on 57758.41297 (23.7 $\mathrm{hr}$ after the GW detection) and detected on seven subsequent overlapping images that in total spanned $1.18 \mathrm{hr}$. It was discovered at R.A. = 138.30789, decl. $=+61.09267$ (09:13:13.89, +61:05:33.6), with an rms positional scatter over the eight images of 0 ". 52 . Only two other extragalactic transients were identified on this night. One is a nuclear transient (ATLAS17ber $=$ PS17em $=$ AT2017aur) coincident with the core of a $r=19.1$ galaxy (SDSS J084004.30 +584703.1 ), which showed variable activity for the next 70 days and is likely central AGN activity. The other (ATLAS17afb) is variability of the known QSO (SDSS J092136.23+621552.1) at $z=1.44746$. Neither of these are likely related to GW 170104 .

In parallel, we observed the central high-probability region with the Pan-STARRS1 (PS1) system (Chambers et al. 2016), similar to our previous GW events. Our joint ATLAS + PS1 strategy is to cover a wide area (several 1000 square degrees) fast with ATLAS (to roughly $19 \mathrm{mag}$ ) and the higher probably region (several 100 square degrees) deeper with PS1 (to roughly $21.5 \mathrm{mag}$ ). We observed a total of 671 square degrees, covering $43.4 \%$ of the probability sky-area to a more sensitive depth $\left(i_{\mathrm{P} 1} \simeq 21.5-22.0\right)$ on the higher probability region (Figure 1$)$. Data were processed, and 115 suspected extragalactic transient objects were detected in the first three days after the event, as described in Smartt et al. (2016b). Of these, we have spectroscopically confirmed 12 as supernovae or AGNs (5 SNIa, 2 SNIb, 3 SNII, 2 and AGNs), with two uncertain classifications dominated by the host galaxy; a summary paper on PS1 findings in LIGO O2 is in preparation. PS1 also detected ATLAS17aeu on $\mathrm{MJD}=57758$ and 57759 in the $i_{\mathrm{P} 1}$ filter, with the first 
Table 1

Photometry and Multiwavelength Fluxes for ATLAS17aeu and Data for GW 170104 and GRB 170105A for Comparison

\begin{tabular}{|c|c|c|c|c|c|}
\hline Telescope & Magnitude/Flux/Fluence & Filter/Waveband & MJD & Position & Ref. \\
\hline GW 170104 & $\cdots$ & $\cdots$ & 57757.42498 & & Abbott et al. (2017) \\
\hline GRB 170105A & & & & See Figure 1 & \\
\hline POLAR & & $80-500 \mathrm{keV}$ & 57758.218137 & & Marcinkowski et al. (2017) \\
\hline AstroSAT CZTI & & $40-200 \mathrm{keV}$ & 57758.218125 & & Bhalerao et al. (2017) \\
\hline Konus-Wind & $2.56_{-0.13}^{+0.18} \times 10^{-6} \mathrm{erg} \mathrm{cm}^{-2}$ & $20 \mathrm{keV}-10 \mathrm{MeV}$ & 57758.218174 & Center: $129.749,+27.904$ & Svinkin et al. (2017) \\
\hline INTEGRAL-SPIACS & & $80 \mathrm{keV}-8 \mathrm{MeV}$ & 57758.218125 & Annulusradius: $34.255_{-14.832}^{+1.812}$ & Marcinkowski et al. (2017) \\
\hline SWASP & $>17.0$ & $r$ & 57758.05948013 & At 17 aeu coords & Steeghs et al. (2017) \\
\hline SWASP & $>16.9$ & $r$ & 57758.09198558 & $\ldots$ & Steeghs et al. (2017) \\
\hline SWASP & $>16.3$ & $r$ & 57758.20992235 & $\cdots$ & Steeghs et al. (2017) \\
\hline PS1 & $17.75 \pm 0.01$ & $i_{\mathrm{P} 1}$ & 57758.389 & $138.30783,+61.09272$ & This paper \\
\hline ATLAS & $18.12 \pm 0.09$ & $c$ & 57758.41297 & $138.30789+61.09267$ & This paper \\
\hline ATLAS & $18.25 \pm 0.11$ & $c$ & 57758.41446 & $138.30789+61.09267$ & This paper \\
\hline ATLAS & $18.26 \pm 0.11$ & $c$ & 57758.42672 & $138.30789+61.0926$ & This paper \\
\hline ATLAS & $18.57 \pm 0.14$ & $c$ & 57758.44191 & $138.30789+61.09267$ & This paper \\
\hline ATLAS & $18.48 \pm 0.13$ & $c$ & 57758.44691 & $138.30789+61.09267$ & This paper \\
\hline ATLAS & $18.34 \pm 0.12$ & $c$ & 57758.44792 & $138.30789+61.09267$ & This paper \\
\hline ATLAS & $18.45 \pm 0.10$ & $c$ & 57758.45499 & $138.30789+61.09267$ & This paper \\
\hline ATLAS & $18.94 \pm 0.17$ & $c$ & 57758.46196 & $138.30789+61.09267$ & This paper \\
\hline PS1 & $18.25 \pm 0.02$ & $i_{\mathrm{P} 1}$ & 57758.464 & $\ldots$ & This paper \\
\hline PS1 & $20.64 \pm 0.10$ & $i_{\mathrm{P} 1}$ & 57759.338 & $\ldots$ & This paper \\
\hline PS1 & $20.71 \pm 0.13$ & $i_{\mathrm{P} 1}$ & 57759.354 & $\ldots$ & This paper \\
\hline PS1 & $20.97 \pm 0.09$ & $i_{\mathrm{P} 1}$ & 57759.539 & $\cdots$ & This paper \\
\hline PS1 & $21.20 \pm 0.12$ & $i_{\mathrm{P} 1}$ & 57759.556 & $\cdots$ & This paper \\
\hline PS1 & $20.88 \pm 0.10$ & $i_{\mathrm{P} 1}$ & 57759.618 & $\ldots$ & This paper \\
\hline Swift-XRT & $2.9 \pm 1.3 \times 10^{-13} \mathrm{erg} \mathrm{cm}^{-2} \mathrm{~s}^{-1}$ & $0.3-10 \mathrm{keV}$ & 57760.03365 & $138.3059+61.0919$ & Evans et al. (2017b) \\
\hline Swift-XRT & $2.5 \pm 0.5 \times 10^{-13} \mathrm{erg} \mathrm{cm}^{-2} \mathrm{~s}^{-1}$ & $0.3-10 \mathrm{keV}$ & 57760.86307 & $\ldots$ & This paper \\
\hline AMI & $336.0 \pm 20.0 \mu \mathrm{Jy}$ & $15.5 \mathrm{GHz}$ & 57760.1667 & Not quoted in GCN & Mooley et al. (2017) \\
\hline Hale & $21.9 \pm 0.3$ & $i$ & 57760.33719 & $\ldots$ & Kasliwal et al. (2017) \\
\hline DCT & $22.08 \pm 0.05$ & $r$ & 57760.45556 & $\cdots$ & Cenko \& Troja (2017) \\
\hline DCT & $21.96 \pm 0.05$ & $i$ & 57760.45556 & $\cdots$ & Cenko \& Troja (2017) \\
\hline DCT & $22.45 \pm 0.05$ & $g$ & 57760.45556 & $\ldots$ & Cenko \& Troja (2017) \\
\hline VLA & $159.0 \pm 9.8) \mu \mathrm{Jy}$ & $6 \mathrm{GHz}$ & 57760.62950 & Not quoted in GCN & Corsi et al. (2017) \\
\hline AMI & $353 \pm 17 \mu \mathrm{Jy}$ & $15.5 \mathrm{GHz}$ & 57761.03 & $\ldots$ & https://4pisky.org/ami-grb/ \\
\hline TNG & $22.5 \pm 0.3$ & $I$ Vega & 57761.09319 & $\cdots$ & Melandri et al. (2017b) \\
\hline Gemini & $22.77 \pm 0.17$ & $r$ & 57761.51968 & $\cdots$ & This paper \\
\hline Swift-XRT & $9.1 \pm 2.8 \times 10^{-14} \mathrm{erg} \mathrm{cm}^{-2} \mathrm{~s}^{-1}$ & $0.3-10 \mathrm{keV}$ & 57762.69006 & $138.3059+61.0919$ & Evans et al. (2017a) \\
\hline Swift-XRT & $<2.6 \times 10^{-13} \mathrm{erg} \mathrm{cm}^{-2} \mathrm{~s}^{-1}$ & $0.3-10 \mathrm{keV}$ & 57764.12551 & $\ldots$ & This paper \\
\hline AMI & $183 \pm 19 \mu \mathrm{Jy}$ & $15.5 \mathrm{GHz}$ & 57765.03 & $\cdots$ & https://4pisky.org/ami-grb/ \\
\hline Swift-XRT & $1.3 \pm 0.6 \times 10^{-13} \mathrm{erg} \mathrm{cm}^{-2} \mathrm{~s}^{-1}$ & $0.3-10 \mathrm{keV}$ & 57765.38889 & $\cdots$ & This paper \\
\hline Swift-XRT & $<2.2 \times 10^{-13} \mathrm{erg} \mathrm{cm}^{-2} \mathrm{~s}^{-1}$ & $0.3-10 \mathrm{keV}$ & 57766.21122 & $\cdots$ & This paper \\
\hline AMI & $142 \pm 19 \mu \mathrm{Jy}$ & $15.5 \mathrm{GHz}$ & 57767.02 & $\cdots$ & https://4pisky.org/ami-grb/ \\
\hline Swift-XRT & $<1.9 \times 10^{-13} \mathrm{erg} \mathrm{cm}^{-2} \mathrm{~s}^{-1}$ & $0.3-10 \mathrm{keV}$ & 57767.37897 & $\cdots$ & This paper \\
\hline AMI & $122 \pm 20 \mu \mathrm{Jy}$ & $15.5 \mathrm{GHz}$ & 57768.03 & $\cdots$ & https://4pisky.org/ami-grb/ \\
\hline AMI & $118 \pm 27 \mu \mathrm{Jy}$ & $15.5 \mathrm{GHz}$ & 57769.03 & $\cdots$ & https://4pisky.org/ami-grb/ \\
\hline AMI & $118 \pm 27 \mu \mathrm{Jy}$ & $15.5 \mathrm{GHz}$ & 57770.03 & $\ldots$ & https://4pisky.org/ami-grb/ \\
\hline Swift-XRT & $5.5 \pm 2.3 \times 10^{-14} \mathrm{erg} \mathrm{cm}^{-2} \mathrm{~s}^{-1}$ & $0.3-10 \mathrm{keV}$ & 57770.50380 & $\cdots$ & This paper \\
\hline AMI & $81 \pm 23 \mu \mathrm{Jy}$ & $15.5 \mathrm{GHz}$ & 57771.03 & $\cdots$ & https://4pisky.org/ami-grb/ \\
\hline AMI & $149 \pm 21 \mu \mathrm{Jy}$ & $15.5 \mathrm{GHz}$ & 57772.04 & $\cdots$ & https://4pisky.org/ami-grb/ \\
\hline AMI & $84 \pm 28 \mu \mathrm{Jy}$ & $15.5 \mathrm{GHz}$ & 57773.04 & $\ldots$ & https://4pisky.org/ami-grb/ \\
\hline AMI & $<81 \mu \mathrm{Jy}$ & $15.5 \mathrm{GHz}$ & 57774.01 & $\cdots$ & https://4pisky.org/ami-grb/ \\
\hline AMI & $<84 \mu \mathrm{Jy}$ & $15.5 \mathrm{GHz}$ & 57775.97 & $\cdots$ & https://4pisky.org/ami-grb/ \\
\hline AMI & $82 \pm 18 \mu \mathrm{Jy}$ & $15.5 \mathrm{GHz}$ & 57777.01 & $\ldots$ & https://4pisky.org/ami-grb/ \\
\hline
\end{tabular}

Note. Data not presented in this paper were released as the GCNs cited. The Swift observations and data were reported in Evans et al. (2017a, 2017b), but we reanalyzed the data in the archive to produce the numbers we quote here. The AMI data are available publicly at the quoted website.

detection just before the ATLAS17aeu point. We recovered the transient in the ATLAS data first because our processing speed for the shallower and lower data rate of ATLAS is faster (by a factor of about 10) than the speed of PS1. All photometry values are reported in Table 1.
We subsequently observed ATLAS17aeu with Gemini North and GMOS (Hook et al. 2004), acquiring both $r$-band imaging and optical spectroscopy on MJD = 57761.595 (2017 January 08.595 UT, 4.2 days after the GW detection). These data were taken with the e2V deep depletion (e2vDD) detector array 
before it was decommissioned in 2017 February (within program GN-2016B-Q-2 PI: Chambers). The spectrum was taken with the R400 grating covering 4490-8878 A, with a 1!"0 slit in seeing conditions of 1 ". 06 . Six separate $900 \mathrm{~s}$ exposures were taken, giving a total of $5400 \mathrm{~s}$ on source. These 2D data were reduced with the IRAF/Gemini package in a standard way, with the arc lamp wavelength calibration checked using sky emission lines. Two methods of spectrum stacking and sky subtraction were used. The first method simply extracted a spectrum from each single $900 \mathrm{~s}$ exposure, with sky subtraction in apertures close to the object. The second involved 2D image subtraction, alignment, and stacking (since the object was shifted spatially during the exposures). Similar results were achieved in each case. The imaging sequence was a set of $6 \times 180 \mathrm{~s}$ exposures that were shifted, aligned, and stacked together. A series of 14 SDSS reference stars within 2 arcmin of the position of ATLAS17aeu were used to photometrically calibrate the stacked image. ATLAS17aeu was clearly detected in the GMOS images at $r=22.77 \pm 0.17$ (Table 1), and a spectrum with a good signal-to-noise ratio revealed a blue featureless continuum $(\mathrm{S} / \mathrm{N} \simeq 10$ per pixel). We applied flux calibration using a standard star with the same instrument setup and a telluric absorption line correction for $\mathrm{O}_{2}$ using an atmosphere model convolved and rebinned to the spectrometer resolution, e.g., as described in Smartt et al. (2015).

When the object had faded, we again used Gemini North and GMOS to take a deep $r$-band image to search for a host galaxy and constrain the redshift of the transient (within program GN-2017A-Q-23 PI: Chambers). On 2017 April 01, we took a series of $13 \times 90 \mathrm{~s}$ images with a midpoint time of MJD = 57844.33 (86.9 days after the GW detection) during the $1170 \mathrm{~s}$ total exposure time. By this time, the new Hamamatsu detector array had been installed in the instrument. We then centered the GMOS slit on the position of ATLAS17aeu and set the slit angle to also cover the closest visible galaxy, which is offset from the source by 1 !" 8 . On the night of MJD $=57845.252$ (2017 April 02, 87.8 days after the GW detection), we took a series of $9 \times 968 \mathrm{~s}$ exposures with the R400 grating, again covering 4490-8878 $\AA$. The observations used the nod-andshuffle mode. Nod-and-shuffle mode interleaves short observations (typically $60 \mathrm{~s}$ each) of the object and the sky without reading out the $\mathrm{CCD}$. The charge is shuffled on the chip into storage regions, resulting in two separate spectra images in a single CCD exposure, one containing the object, and the other containing only the sky. The two spectra images are then subtracted from each other, which helps remove strong sky emission residuals, leaving increased Poisson noise at the position of the skylines. As the IRAF/Gemini package does not yet function with the Hamamatsu detector array, we employed manual reductions for both the imaging and spectroscopic modes. After subtracting the spectra images from each other, we then shifted and stacked the nine exposures together. The results are described in Section 4.

In addition, we used Keck II and the DEIMOS spectrometer to take spectra with the same slit position as set for the GMOS spectra on the night of MJD $=57870$ (2017 April 27, 113 days after the $\mathrm{GW}$ detection). We took $3 \times 1200 \mathrm{~s}$ with the R600ZD grating and a 1". 2 slit, giving a spectral range of 5550-9839 (with the OG550 blocking filter) and a resolution of $4.5 \AA$ at the center of the range. DEIMOS is an eight-detector array, and ATLAS17aeu fell on chips 3 and 7 (Video Input 6 and 14). The data of these two detectors were debiased using overscan, and they were flat-fielded and extracted in standard fashion. After correcting for the 2D distortion on these chips, we forced an extraction of the weak signal close to the position of ATLAS17aeu. The results of the imaging and spectroscopy are described in Section 4.

ATLAS17aeu immediately stood out as an unusual and rare transient in the ATLAS and Pan-STARRS1 data streams that occurred within one day of GW 170104 (Tonry et al. 2017). The decay rate of $0.71 \mathrm{mag} \mathrm{hr}^{-1}$ is faster than typical CV decline (Bailey 1975) and slower than M-dwarf flares (Berger et al. 2013). The PS1 images confirmed the fading on the second night of observations when it had faded below the ATLAS detection limit. During the first year of general ATLAS surveying, we typically took five dithered exposures per night, separated across approximately $1 \mathrm{hr}$. PS1 has been observing in a set of quad exposures each night, separated by about 15 minutes each, since 2015 . In both these surveys, we find many supernovae, M-dwarf and CV-like candidates (Tonry et al. 2016), but fast transients that decline with this rate are rare (see Section 5 for a further discussion of rates). Therefore we immediately released this information (in Tonry et al. 2017) to the broad LIGO-Virgo EM follow-up effort (see Abbott et al. 2016a). This instigated a series of multiwavelength observations, which we summarize in the next section.

\section{Multiwavelength Observations and GRB 170105A}

ATLAS17aeu was observed in both the X-ray by Swift (Evans et al. 2017b) and in the radio by the Arcminute MicroKelvin Imager (AMI; Mooley et al. 2017) and the Very Large Array (VLA Corsi et al. 2017). Evans et al. (2017b) reported an X-ray source with Swift $(0.3-10 \mathrm{keV}$; R.A., decl. $=138.3059$, $+61.0919)$ on $\mathrm{MJD}=57760.034$ (2.6 days after the $\mathrm{GW}$ detection) at 4 !" $1 \pm 6$ ". 3 from the position of ATLAS17aeu. We downloaded the Swift X-ray Telescope (XRT) images from the archive and measured fluxes using the HEASARC sosta task on eight separate epochs from $\mathrm{MJD}=57760.03365$ to 57770.50380. We used $N_{\mathrm{H}}=4.6 \times 10^{20} \mathrm{~cm}^{-2}$ and a photon index of $\Gamma=1.6$ (as in Evans et al. 2017a) to convert the measured counts $\mathrm{s}^{-1}$ into $\mathrm{erg} \mathrm{cm}^{-2} \mathrm{~s}^{-1}$ using HEASARC WebPIMMS. A subsequent enhanced position was published on the Swift website as R.A., decl. = 138.30789, 61.09263 with an error radius of 2.2 arcsecond (90\% confidence), which is also consistent with ATLAS17aeu. The unabsorbed fluxes over the $0.3-10 \mathrm{keV}$ range are reported in Table 1 and plotted in Figure 2. The HEASARC tool xselect was used to extract XRT images in different energy range frames from which the photons were counted. A histogram of the energy distribution of individual photons from the five epochs with X-ray detection is shown in Figure 3. The values cluster around $1 \mathrm{keV}$, consistent with a soft $\mathrm{X}$-ray source. Two radio detections were reported, the first by Mooley et al. (2017) with the AMI-LA (Arcminute Microkelvin Imager Large Array) at $15 \mathrm{GHz}$ on MJD $=57760.04$ (2.6 days after the GW detection) and shortly later by Corsi et al. (2017) with the VLA at $6 \mathrm{GHz}$ in an observation covering $\mathrm{MJD}=57760.58796$ to 57760.62950 . The AMI results are publicly available ${ }^{8}$ and are listed in Table 1.

A gamma-ray burst was discovered independently of all these data by POLAR (GRB 170105A; Marcinkowski et al. 2017) with a discovery time that could imply a link between the

\footnotetext{
https://4pisky.org/ami-grb/
} 

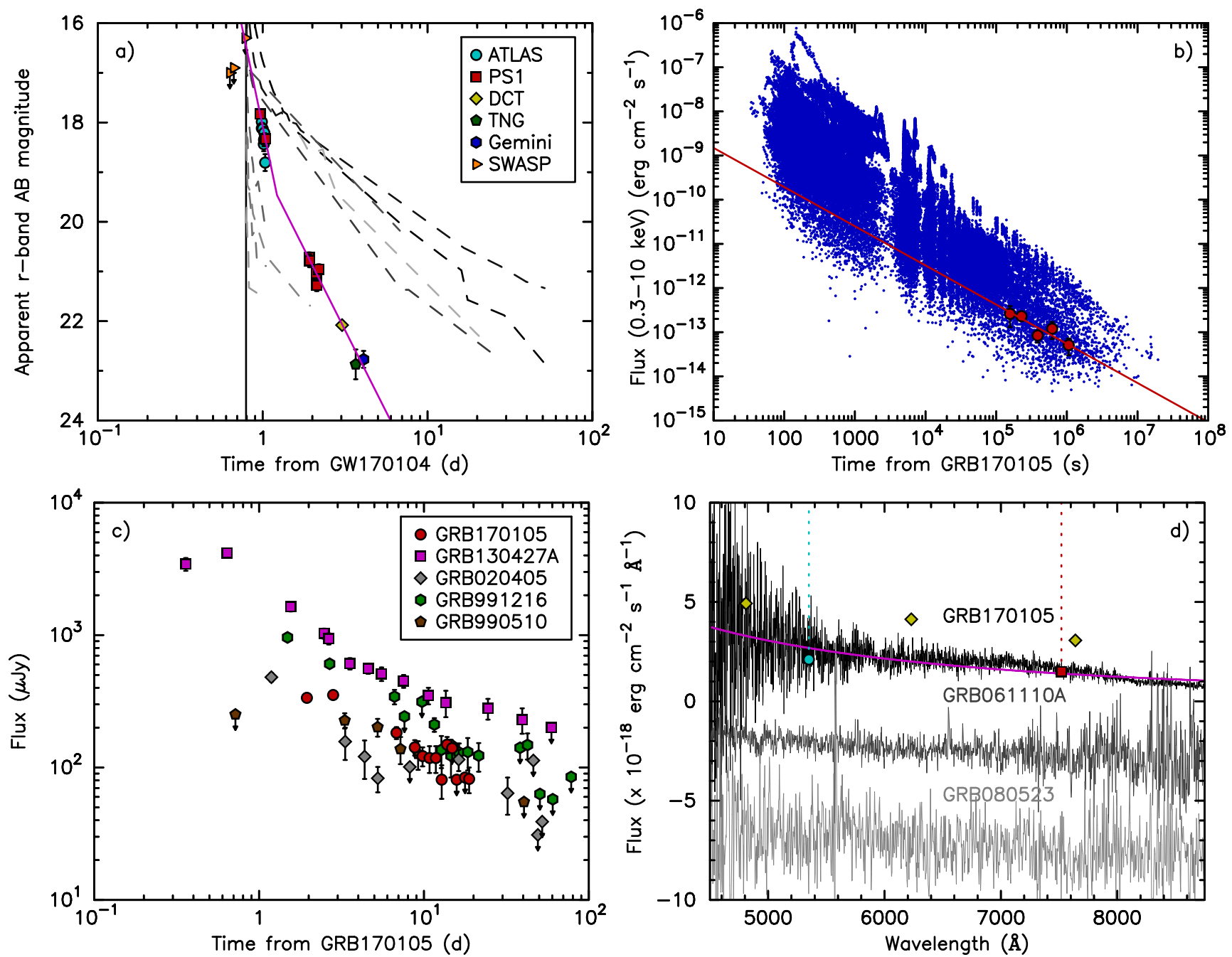

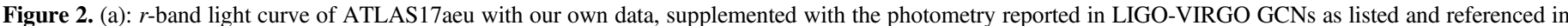

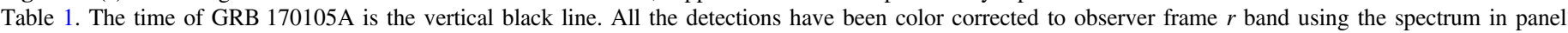

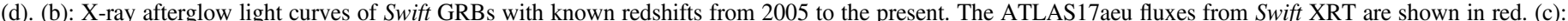

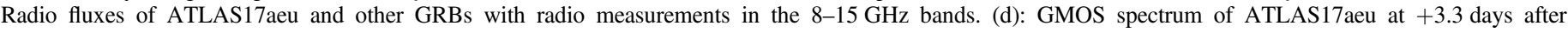

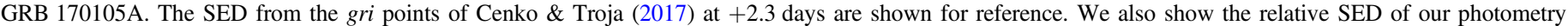

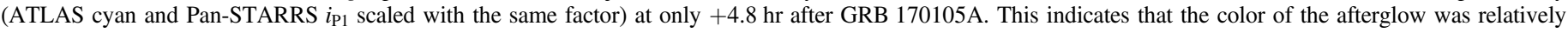
constant over the first three days.

GRB and ATLAS17aeu (as initially proposed by Kasliwal et al. 2017), or perhaps linking either of the events to GW 170104. The GRB was also detected by AstroSat CZTI (Sharma et al. 2017), Konus-Wind, and INTEGRAL SPI-ACS (Svinkin et al. 2017). The properties of the GRB are summarized in Table 1. The GRB fluence from Konus-Wind was $2.56_{-0.13}^{+0.18} \times 10^{-6} \mathrm{erg} \mathrm{cm}^{-2}$, within the energy range $20 \mathrm{keV}-$ $10 \mathrm{MeV}$ (Svinkin et al. 2017). The signal in the three energy bands of Konus-Wind ${ }^{9}$ shows no flux in the hardest band $300-1160 \mathrm{keV}$, but significant detections in the 18-70 and $70-300 \mathrm{keV}$ channels. We estimated the $T_{50}$ and $T_{90}$ durations (the time intervals containing $25 \%-75 \%$ and $5 \%-90 \%$ of the total fluence) from the available Konus-Wind light curve and found $T_{50}=1.1 \mathrm{~s}, T_{90}=2.8 \mathrm{~s}$ in the publicly available integrated energy bandpass of $50-200 \mathrm{keV} .{ }^{10}$ Bhalerao et al. (2017) reported a $T_{90}=15 \pm 1 \mathrm{~s}$, and with no signal above $100 \mathrm{keV}$, they suggest a classification of a long, soft GRB.

\footnotetext{
9 http://www.ioffe.ru/LEA/GRBs/GRB170105_T22450/

10 https://gcn.gsfc.nasa.gov/konus_grbs.html
}

While we measured $T_{90}=2.8 \mathrm{~s}$ in the integrated energy bandpass of $50-200 \mathrm{keV}$ (and $T_{50}=1.1 \mathrm{~s}$ ), it appears that there is a flux over a longer duration of about $20 \mathrm{~s}$ in the softest channel only (18-70 keV Svinkin et al. 2017). This is visible in the online plot of the GRB duration (in the low time-resolution plot), but is not captured in the high time-resolution data files. Svinkin et al. (2016) presented the second Konus-Wind sample of short GRBs, and in defining the sample, they adopted $T_{50}<0.6 \mathrm{~s}$. This is somewhat of an arbitrary cutoff in order to define a relatively clean sample of short bursts, which could be further sub-classified. However, Svinkin et al. (2016) more quantitatively defined two Gaussian distributions in the hardness-duration plane for 1143 Konus-Wind bursts. The long $20 \mathrm{~s}$ duration of GRB 170105A in the soft $\gamma$-ray channel puts it comfortably within the long GRB Gaussian distribution and outside the $3 \sigma$ contour that defines the short, hard GRBs. The hardness ratio from Konus-Wind is defined as the ratio of counts in the $G 3$ to $G 2$ bands $\left(H_{2}\right)$. These quantitative fluxes are not available publicly yet, but since there is no flux in the $300-1160 \mathrm{keV}$ range (roughly band G3), it is 


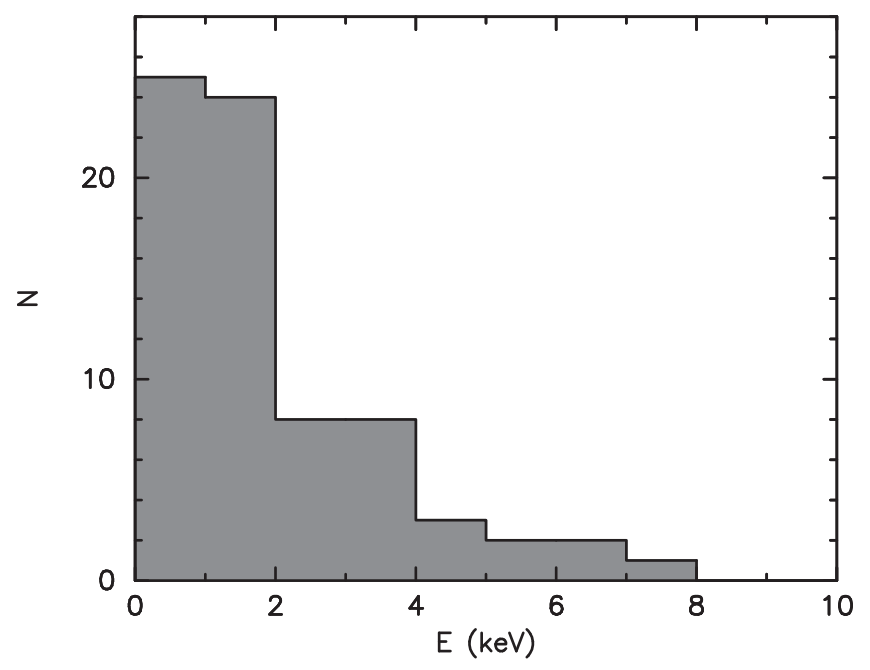

Figure 3. Histogram of the energy distribution of individual photons from the five epochs with XRT X-ray detection.

likely that this GRB is long and soft. The properties of this GRB in context with the Swift, Fermi, and Konus-Wind detected GRBs is further discussed in Section 5.

The detections by Konus-Wind, and INTEGRAL (SPI-ACS) allowed triangulation of the signal, resulting in a sky annulus that has a radius of $34^{\circ} .255$ (and is $16^{\circ} .644$ wide) centered on R.A. $=$ $129^{\circ} .749\left(08^{\mathrm{h}} 38^{\mathrm{m}} 60^{\mathrm{s}}\right)$ and decl. $=+27^{\circ} .904\left(+27^{\mathrm{d}} 54^{\mathrm{m}} 14^{\mathrm{s}}\right)$, as reported in Svinkin et al. (2017). ATLAS17aeu is $34^{\circ} .040$ from the center and within the annulus, and therefore the positions are consistent. Figure 1 shows the sky map from LIGO for GW 170104 and the likely GRB 170105A annulus from (Svinkin et al. 2017) together with the position of ATLAS17aeu. A joint AstroSat CZTI + IPN localization analysis showed ATLAS17aeu to be spatially consistent with the detection (Bhalerao et al. 2017).

Therefore we have three distinct astrophysical events, GW 170104, ATLAS17aeu, and GRB 170105A, that are spatially coincident within the uncertainties and are temporally coincident within $24 \mathrm{hr}$. We now consider the possibility that ATLAS17aeu is related to either of these transients and the likelihood that all three are physically related.

\section{Host Galaxy and Redshift Constraints for ATLAS17aeu}

The relative volumetric rates of GRBs, GRB-like afterglows, and GW sources can provide information for a discussion of the probability of coincidences. Therefore an identification of the host galaxy and redshift determination of ATLAS17aeu is desirable. The initial GMOS spectrum of ATLAS17aeu, when it was fading at $r=22.77$, shows a blue featureless continuum (Figure 2). There are no obvious absorption lines such as $\mathrm{Mg}$ II (from interstellar matter (ISM) absorption in the host) and no strong nebular emission lines (e.g., $\mathrm{H} \alpha, \mathrm{O}[\mathrm{III}]$ ) from star formation in the host. The blue featureless continuum can be fit with a power law with index $\alpha=-1.92$. This is not unlike other GRB afterglow spectra, for example from the extensive study of Fynbo et al. (2009). For some of these examples, a definitive redshift has proven to be difficult to obtain. Two examples are shown in Figure 2, GRB 060110A and GRB 080523 from Fynbo et al. (2009). The shape of the spectral energy distribution (SED) of ATLAS17aeu from the Gemini spectrum (which is +3.3 days after GRB 170105A) is consistent with the color inferred from the ATLAS cyan and the Pan-STARRS $i_{\mathrm{P} 1}$ photometry at just $+4.8 \mathrm{hr}$ after the GRB.
The SED from the Discovery Channel Telescope photometry (at +2.3 days after GRB 170105A) by Cenko \& Troja (2017) also shows the same blue slope. This indicates that the optical SED of ATLAS17aeu stayed relatively constant for the three days over which it was observed, with a power-law index $\alpha=-1.92$.

A montage of our images is shown in Figure 4. We originally noted in Tonry et al. (2017) that ATLAS17aeu is 23 arcsec from the face-on, bright, spiral galaxy SDSS J091312.36+610554.2, which has a spectroscopic redshift of $z=0.19900 \pm 0.00004$. This implies a luminosity distance of $990 \mathrm{Mpc}$, so if ATLAS17aeu were related, it would be at a projected distance of $75 \mathrm{kpc}$ from this galaxy (see Figure 4). This luminosity distance is consistent with the LIGO distance to GW 170104, which suggests a $10-90$ percentile probability of $520-1010 \mathrm{Mpc}$ in this direction (from the analysis of Singer et al. 2016). However, our deep GMOS imaging reveals the environment of ATLAS17aeu in more detail. There is a faint galaxy exactly at the position of ATLAS17aeu in the Gemini image of 2017 April 01, which we label "Galaxy A" in Figure 4. With aperture photometry, we measure $r=25.59 \pm 0.16$. There is also a brighter extended source that is 1 !'8 southeast of the position of ATLAS17aeu (R.A. = 9:13:14.120, decl. $=+61: 05: 32.60)$, for which we measure $r=24.44 \pm 0.09$. We label this "Galaxy B," and it is clearly visible in the earlier Gemini image (from 2017 January 08) resolved from ATLAS17aeu. In this image we measure $r=24.11 \pm .09$, with the difference between the two arising from the choice of aperture that is required to minimize contamination from the bright flux of ATLAS17aeu. Galaxy B is almost certainly the object report by Melandri et al. (2017a) at $r=24.23 \pm 0.2$, which they proposed as a host. Our GMOS images now show that this is offset and resolved from the position of ATLAS17aeu. It is possible that Galaxies A and B are physically linked and could either be a merging or disturbed system, and a redshift for both is desirable.

We placed the slits of GMOS and DEIMOS (see Section 2 for details of these deep spectra) across these two galaxies as illustrated in Figure 4 in search of any emission lines of either that would provide a redshift for ATLAS17aeu. There is no obvious emission line detected at the spatial positions of Galaxies A or B. The slit passed through two brighter galaxies, for which we measure redshifts $z=0.3487$ for the star-forming SDSS J091318.49+610512.6 and $z=0.2765$ for the earlytype galaxy SDSS J091310.39+610548.6 (labeled in Figure 4 for completeness). In the Gemini GMOS spectrum (for which nod-and-shuffle was used), faint continuum is present for Galaxy B, whereas no apparent signal is detected at the position of Galaxy A (see Figure 5). We are confident in the location of Galaxy B on the 2D spectral images from the spatial consistency of its position and measured offsets from the $z=0.3487$ and $z=0.2765$ bright sources located in the slit. In the first GMOS spectrum of ATLAS17aeu, a weak, possible emission line at $7860.3 \pm 0.5 \AA$ is illustrated in the inset panel of Figure 5. If this were confirmed, it could potentially be $\mathrm{H} \alpha$ from Galaxies A or B at $z=0.199$. The feature is visible in two different extraction methods, either extracting and combining the six individual spectra, or combining and sky subtracting the 2D frames and then extracting the object. It has a FWHM of $5.7 \pm 0.7 \AA$, which is narrower than the skylines measured on the frames $(6-7.6 \AA)$. No spatially extended flux is visible in the $2 \mathrm{D}$ spectral frame at this position. An excess in flux is only marginally visible in the 2D spectrum of 

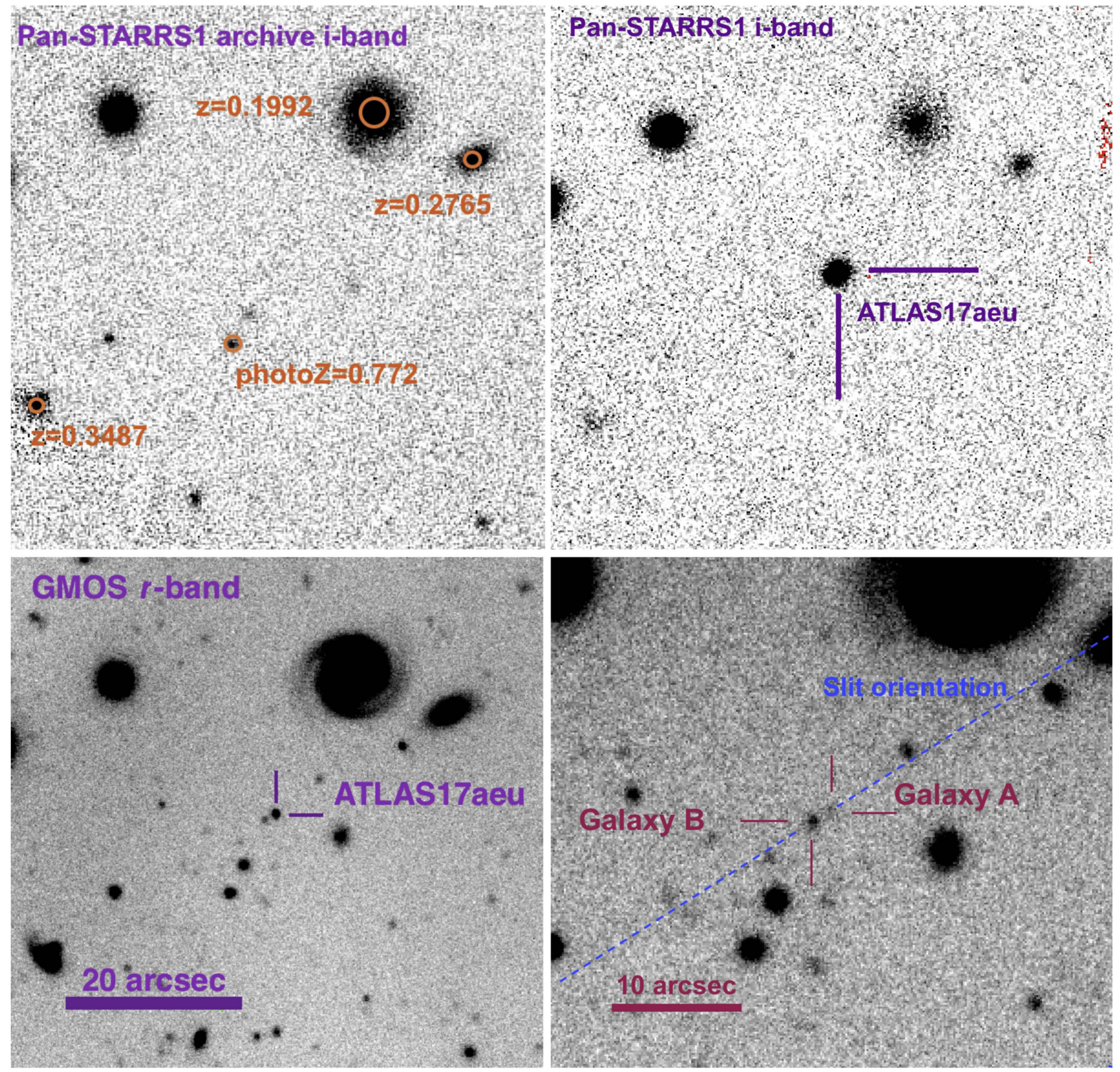

Figure 4. Top left panel: Pan-STARRS1 $i_{\mathrm{P} 1}$-band archive image (Chambers et al. 2016), showing no host down to $i_{\mathrm{P} 1} \simeq 23$ at the position of ATLAS17aeu. The redshifts of neighboring galaxies are shown with the $z=0.1992$ and the $z_{\text {photo }}=0.772$ values from SDSS DR12 and the other two from our GMOS and Keck spectra. Top right panel: Pan-STARRS1 detection of ATLAS17aeu on $57758.389\left(i_{\mathrm{P} 1}=17.75\right)$ Bottom left panel: Gemini-North GMOS image of ATLAS17aeu $(r$ band $)$ on MJD $=57761.51968$, showing the object at $r=22.77$ Bottom right panel: Deep Gemini-North GMOS image of ATLAS17aeu $(r$ band) at 86 days after discovery. The host of ATLAS17aeu is Galaxy A, and the nearby brighter Galaxy B (1"! 8 separation) is also labeled. The slit orientation for the Gemini GMOS and Keck

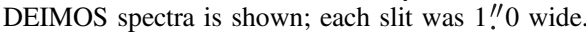

ATLAS17aeu at this position (which would be Galaxy A). However, we find no confirmation of the line in the GMOS or DEIMOS deep spectra taken later (Figure 5) and therefore cannot confirm a redshift for either Galaxy A or Galaxy B. A faint continuum for each of Galaxies A and B is visible in the Keck 2D images, and we forced extraction of the signal using the trace of the bright $z=0.2765$ galaxy. Since slit nodding or shifting was not used in the Keck spectra, the red part is dominated by skyline noise. We manually snipped out the lines and rebinned the 1D flux-calibrated spectra to $20 \AA$ per pixel dispersion. For this coarse spectrum, the overall SED should be reliable. We then applied synthetic photometry and scaling to bring the continuum into line with $r=24.1$ and $r=25.6$ for Galaxies B and A, respectively. Figure 5 shows that Galaxy B is bluer than $\mathrm{A}$, but no reliable redshift could be determined. Galaxy A looks relatively flat in its SED, while Galaxy B clearly does have a rising blue continuum (which we also see in the Gemini spectrum). There is no clear sign of the $4000 \AA$ break, for example, in either galaxy. This could mean that $z \lesssim 0.25$, but it is not a definitive statement given the signal-tonoise ratio and wavelength coverage of the data. We may be seeing either the rising optical continuum of a low-redshift galaxy or the rising UV (2000-3000 ̊) continuum of an Sb-Sd star-forming galaxy. The fact that we see no strong absorption 


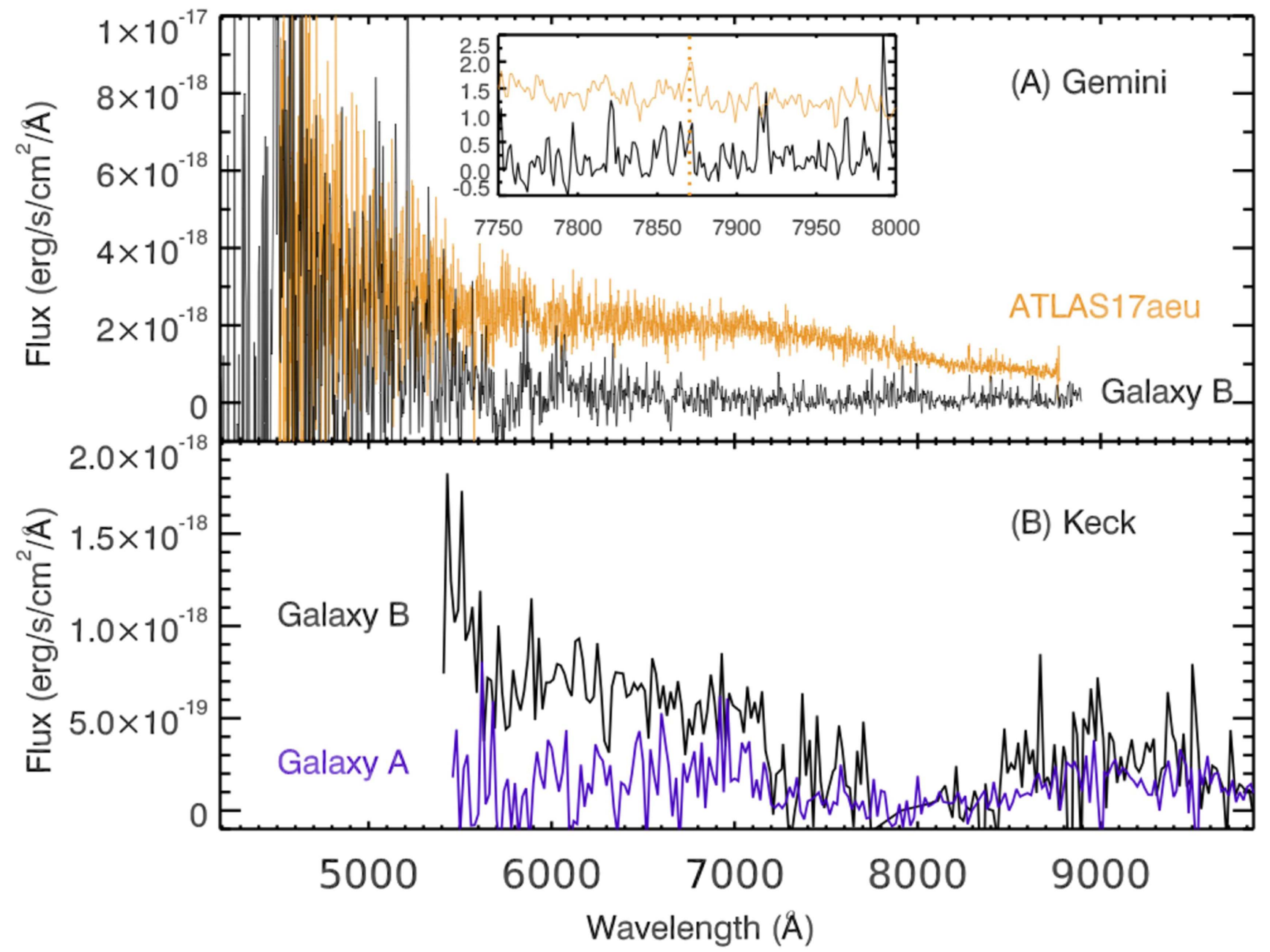

Figure 5. Top panel: 2D spectral image from Gemini + GMOS taken when ATLAS17aeu had faded, in order to search for any residual host galaxy emission lines. The faint trace of Galaxy B is labeled, and there is no discernible flux at the position of Galaxy A. Bottom panel: extracted flux-calibrated spectra from GMOS and Keck. In panel (A) the ATLAS17aeu spectrum from Gemini on 57761 (3.3 days after GRB 170105A) is plotted in orange. The later deep spectrum of extraction of Galaxy B (shown in the 2D image) is shown in black. Galaxy A is not clearly visible in the GMOS spectra, although a forced extraction can be made. The possible emission line in the ATLAS17aeu spectrum at $7870.3 \AA$ is not clearly visible above the noise in the deeper 2D spectral image or in the extracted spectrum, as indicated in the inset. In panel (B) the extracted Keck DEIMOS spectra of Galaxies A and B are shown. The skylines dominate the red and were manually snipped out, after which the spectra were rebinned to $20 \AA$ A per pixel dispersion. Synthetic photometry was used to scale the flux level to match the measured $r$-band magnitudes in the difference image.

that would be due to the Ly $\alpha$ forest in the first GMOS spectrum of ATLAS17aeu provides a robust upper limit to the redshift of $z \lesssim 2.9$ (as has been done for other GRBs in Fynbo et al. 2009). As far as we can tell with these data, there is no clear evidence of either being high redshift $(z \gtrsim 1)$. At the position of ATLAS17aeu (Galaxy A, where we see no continuum flux in the Gemini spectrum), the $3 \sigma$ upper limit for an emission line is (3-4) $\times 10^{-18} \mathrm{erg} \mathrm{s} \mathrm{cm}^{-2}$ depending on where the measurement is made. In the Gemini spectrum of Galaxy B, the limit is around $1 \times 10^{-17} \mathrm{erg} \mathrm{s} \mathrm{cm}^{-2}$. We chose to make these measurements on the Gemini spectrum only because of the superior sky-subtraction methods employed during observing and reductions.

Therefore we are left with the conclusion that ATLAS17aeu has a very faint host galaxy at $r=25.59 \pm 0.16$ that is offset by 1 " 8 from another galaxy at $r=24.27 \pm 0.09$. We have no firm redshift constraints for either, nor can we say whether they are physically (kinematically) linked. The sample of host galaxies of 46 GRBs from Savaglio et al. (2009), which extends out to redshift $z \simeq 2$, indicates that about $13 \%$ (6 out of 46) are located in galaxies $r=25$ or fainter. Krühler et al. (2015) shows that for GRBs with $z \lesssim 0.5$, there are no objects with host galaxies fainter than $r \sim 25$, but that they are relatively common beyond $z \gtrsim 1$. If ATLAS17aeu were at the redshift of GW $170104\left(z=0.18_{-0.07}^{+0.08}\right.$ or $\left.D_{\mathrm{L}}=880_{-360}^{+460} \mathrm{Mpc}\right)$, then it would be only $g \simeq-14.2 \mathrm{mag}$, which is very faint even for GRB host galaxies. When we assume this redshift, it would imply a limit on the $\mathrm{H} \alpha$ luminosity $L_{\mathrm{H} \alpha} \leqslant 4.0_{-2.6}^{+5.4} \times$ $10^{38} \mathrm{erg} \mathrm{s}^{-1}$ and a limit on the star formation rate (SFR) of SFR $\leqslant 0.003_{-0.002}^{+0.004} M_{\odot} \mathrm{yr}^{-1}$. Of course, we have no firm redshift constraints other than that it is $z \leqslant 2.9$, which would correspond to $L_{\mathrm{H} \alpha} \leqslant 3.2 \times 10^{41} \mathrm{erg} \mathrm{s}^{-1}$ or an SFR limit of SFR $\leqslant 2.5 M_{\odot} \mathrm{yr}^{-1}$. In conclusion, we do not have a measured redshift for the host of ATLAS17aeu, but the faintness of the detected galaxy and the deep limits on $\mathrm{H} \alpha$ emission imply that a high-redshift GRB origin $(z \gtrsim 1)$ for ATLAS17aeu would be compatible with the characteristics of the known population of long, soft GRBs. If ATLAS17aeu were associated physically 
Table 2

Sky Rates of GRBs and Their X-Ray and Optical Afterglows

\begin{tabular}{|c|c|c|}
\hline Object & Rate & Reference \\
\hline Fermi GRBs (GBM) & 238 per year & von Kienlin et al. (2014) \\
\hline Swift GRBs & 99 per year & http://www.grbcatalog.org/ \\
\hline Fermi + Swift & 275 per year & See Section 4 \\
\hline Fraction of Swift GRBs with X-ray afterglow & 0.95 & Evans et al. (2009) \\
\hline Fraction of Swift GRBs with $m<17$ optical afterglow & 0.3 & Cenko et al. (2009) \\
\hline Fraction of GRBs in $r \geqslant 25$ hosts & 0.13 & Savaglio et al. (2009) \\
\hline Fraction of LGRBs within $z<0.25$ & 0.025 & Berger (2014) \\
\hline Fraction of all (short+long) sGRBs within $z<0.25$ & 0.158 & Berger (2014) \\
\hline
\end{tabular}

Note. The top three rows are all sky rates per year. The bottom five rows are relative fractions.

with GW 170104, then differences between it and the known GRB population might be expected.

\section{Discussion and Analysis}

We begin by investigating the scenario according to which ATLAS17aeu is indeed the optical, X-ray, and radio afterglow of GRB 170105A. The X-ray source and the radio source are to a very high degree of probability the counterparts of ATLAS17aeu. The detection times of the three transients are listed in Table 1 in decimal MJDs and given here in UT times to the nearest second: GW 170104 at 2017 January 04 10:11:58 (Abbott et al. 2017), GRB 170105A at 2017 January 05 06:14:07 (Marcinkowski et al. 2017; Sharma et al. 2017), and ATLAS17aeu at 2017 January 05 09:54:40. The Swift X-ray transient is within 4". 1 (within the $1 \sigma$ error bars) of ATLAS17aeu. Although the radio detections do not state their positional uncertainty, the radio instruments have pointing uncertainties of about 5 "' 0 , indicating their likely positional coincidence. The probability that either the radio or the X-ray transient is coincident with ATLAS17aeu to with 5 " 0 is $\approx 10^{-10}$. Trivially, we must assume that these transients are from the same object, ATLAS17aeu.

Figure 2 shows the optical and X-ray light curves of ATLAS17aeu in comparison with other GRB afterglows. The decline rate in the optical is a broken power law with indexes $\alpha_{1}=1.20 \pm 0.02$ and $\alpha_{2}=0.82 \pm 0.02$, which is compatible with other known GRB afterglows from Cenko et al. (2009) and may offer insight into the circum-burst medium. The X-ray light curve for Swift XRT is shown in comparison with 297 GRBs with known redshifts, and again, it sits within the expected locus of points. GRBs show a trend between X-ray flux and $\gamma$-ray fluence, albeit with a broad spread of more than one order of magnitude (see Figure 12 of Evans et al. 2009). The fluence of GRB 170105A $\left(2.56_{-0.13}^{+0.18} \times 10^{-6} \mathrm{erg} \mathrm{cm}^{-2}\right.$; Svinkin et al. 2017) should be compared with the estimate of X-ray flux at $11 \mathrm{hr}$ after burst. Figure 2 indicates that this would be on the order of $10^{-12}$ erg cm $\mathrm{cm}^{-1}$ if we extrapolate back to $\sim 4 \times 10^{4} \mathrm{~s}$ after the GRB. The ratio of X-ray flux to $\gamma$-ray fluence thus comfortably sits in the observed range of GRB afterglows. Long GRBs show a characteristic range of optical to X-ray flux when both are estimated at $t=1000 \mathrm{~s}$ from the burst (Cenko et al. 2009). For ATLAS17aeu we estimate $F_{\mathrm{opt}}=660 \mu \mathrm{Jy}$ and $F_{\mathrm{x}}=11 \mu \mathrm{Jy}$, after correcting the $r$-band extrapolated point for Galactic ISM extinction and the extrapolated XRT data for Galactic H I column density (and assuming an energy band of $1 \mathrm{keV}$, see Figure 3). This falls within the broad range of the Cenko et al. (2009) sample and is within the spectral index $0.5<\beta<1.25$ expected for GRB afterglows. While these numbers are compatible, we need to consider the rates of GRB more quantitatively to determine the probability of an association of ATLAS17aeu with GRB 170105A.

We now calculate the probability that the $\gamma$-ray burst is independent of ATLAS17aeu (i.e., just a chance coincidence). We use Poisson statistics, where the probability of an occurrence of $n$ events is given by

$$
P(n)=\frac{e^{-\lambda} \lambda^{n}}{n !},
$$

where $\lambda$ is the expectation value. The value of $\lambda$ is the product of a number of factors given by the rate of each

$$
\lambda=\prod_{i=1}^{k} r_{i}
$$

The rates we discuss in this section are listed for reference in Table 2. In this case, we derive the Poisson probabilities of obtaining one or more random coincidences, which simplifies Equation (1) to

$$
p=1-e^{-\lambda}=1-e^{-\prod_{i=1}^{k} r_{i}} .
$$

To determine the time coincidence, we take as a lower bound the SWASP observation at MJD = 57758.20992235 (Steeghs et al. 2017) and as an upper bound the first PS1 observation (to an approximate depth of $21.5 \mathrm{mag}$ ) at 57758.38892. GRB 170105A occurred at 57758.218137. Therefore we have a time coincidence window of $(-0.01,0.17)$ days. The second Fermi GBM catalog (von Kienlin et al. 2014) and the Swift catalog ${ }^{11}$ (Evans et al. 2009) indicate rates of 238 and 99 GRBs per year in each facility. However, there is obviously overlap between the two samples. The Fermi GBM catalog indicates that $26 \%$ of their sources were also detected by Swift, therefore we estimate a total sky rate of Fermi GBM + Swift of 275 per year. For a GRB rate of 275 per year $\left(0.75\right.$ per day; $\left.r_{1}=0.75\right)$ and a time window of 0.18 days $\left(r_{2}=0.18\right)$, this results in a probability of obtaining one or more random coincidences of $p=0.13$.

The positional coincidence from the IPN triangulation of Svinkin et al. (2017) means that we should use the positional annulus for the GRB 170105A, which covers 2901.5 square degrees, resulting in a probability of 0.07 compared to all sky (i.e., $r_{3}=0.07$ ). Combining these probabilities, we obtain a probability of $1 \%$ that GRB 170105 A was simply a chance

\footnotetext{
11 http://www.grbcatalog.org/
} 
coincidence with ATLAS17aeu. $\left(p=1-e^{r_{1} r_{2} r_{3}}=0.01\right)$. In other words, a chance coincidence of ATLAS17aeu and GRB 170105A can be ruled out with 99\% (2.6 $\sigma$ ) confidence.

This calculation assumes, of course, that ATLAS17aeu has properties that lead us to hypothesize that an association is plausible. We are justified in this since we find that the X-ray, optical, and radio detections are similar to GRB afterglows. The three energy bands of Konus-Wind show no detected flux in the hardest channel of $300-1160 \mathrm{keV}$. This, combined with the $T_{90}$ measurements of POLAR and AstroSat CZTI of between 2 and $15 \mathrm{~s}$ and the soft extended $\gamma$-ray flux (around $20 \mathrm{~s}$ duration) detected by Konus-Wind (18-70 keV), would classify GRB $170105 \mathrm{~A}$ as a very soft GRB (as discussed above in Section 3) with extended emission. In Figure 6 we place GRB 170105A in the locus of the Swift and Konus-Wind bursts with the measured $T_{50} \sim 1.1$ and with an approximate upper limit of $\alpha<-2$. While we are aware that $T_{50}$ is larger in the softest channel (only), we do not have access to those data to make a quantitative measurement, and therefore we plot the specific measurement we can make on the integrated $50-200 \mathrm{keV}$ data. Its location is not particularly unusual in the broad population of GRBs, although how soft the spectrum is remains to be reported by the Konus-Wind team. Since the redshift is unknown, the arrow in the $T_{50} /(1+z)$ versus $E_{\text {iso }}$ plane represents the possible position of ATLAS17aeu from $z=0.18$ (were it to be associated with GW 170104) and $z=2.9$, which is our upper limit from the lack of $\operatorname{Ly} \alpha$ absorption in the Gemini afterglow spectrum.

Anderson et al. (2014) reported radio observations of the GRB130427A with the Arcminute Mircrokelvin Imager (AMI) at a frequency of $15.7 \mathrm{GHz}$ between 0.36 and 59.32 days. At a redshift of $z=0.340$, it had a flux of $1300 \mu \mathrm{Jy}$ at +2 days, which is four times brighter than ATLAS17aeu at the same epoch (with respect to the GRB in each case), as shown in Figure 2. Anderson et al. (2014) compiled early radio observations of GRBs and illustrated that the brightness temperatures can be estimated from

$$
T_{\mathrm{b}}=1.153 \times 10^{-8} d^{2} F_{\nu} \nu^{-2} t^{-2}(1+z)^{-1},
$$

where $d$ is the distance (in $\mathrm{cm}$ ), $F_{\nu}$ is the flux (Jy), $\nu$ is the observed frequency in $\mathrm{Hz}$, and $t$ is time (s) since the GRB. Anderson et al. (2014) further discuss that a minimum Lorentz factor of a jet (assuming the standard GRB scenario) can be estimated from $T_{b} / T_{B}=\Gamma^{3}$, where the maximum brightness temperature is the inverse-Compton limit $T_{B} \sim 10^{12} \mathrm{~K}$ (as in Galama et al. 1999). For ATLAS17aeu to have a comfortably high Lorentz factor of $\Gamma>5$ (the minimum found in Anderson et al. 2014), the redshift must be $z \gtrsim 1$. Therefore, one explanation is that ATLAS17aeu is the X-ray, optical, and radio afterglow of GRB 170105A, and that the GRB is part of the known population of long, soft GRBs between redshift $1 \lesssim z \lesssim 2.9$. All the data we have would be consistent with this explanation, including the low probability of a chance coincidence of the two ( $p=0.01$ as discussed above).

We assume then that ATLAS17aeu is the optical, X-ray, and radio afterglow of GRB 170105A and now consider the rate of GRBs to determine the Poisson probability of coincidence of this gamma-ray, X-ray, optical, and radio transient with GW 170104. The GRB 170105A was approximately $t=1$
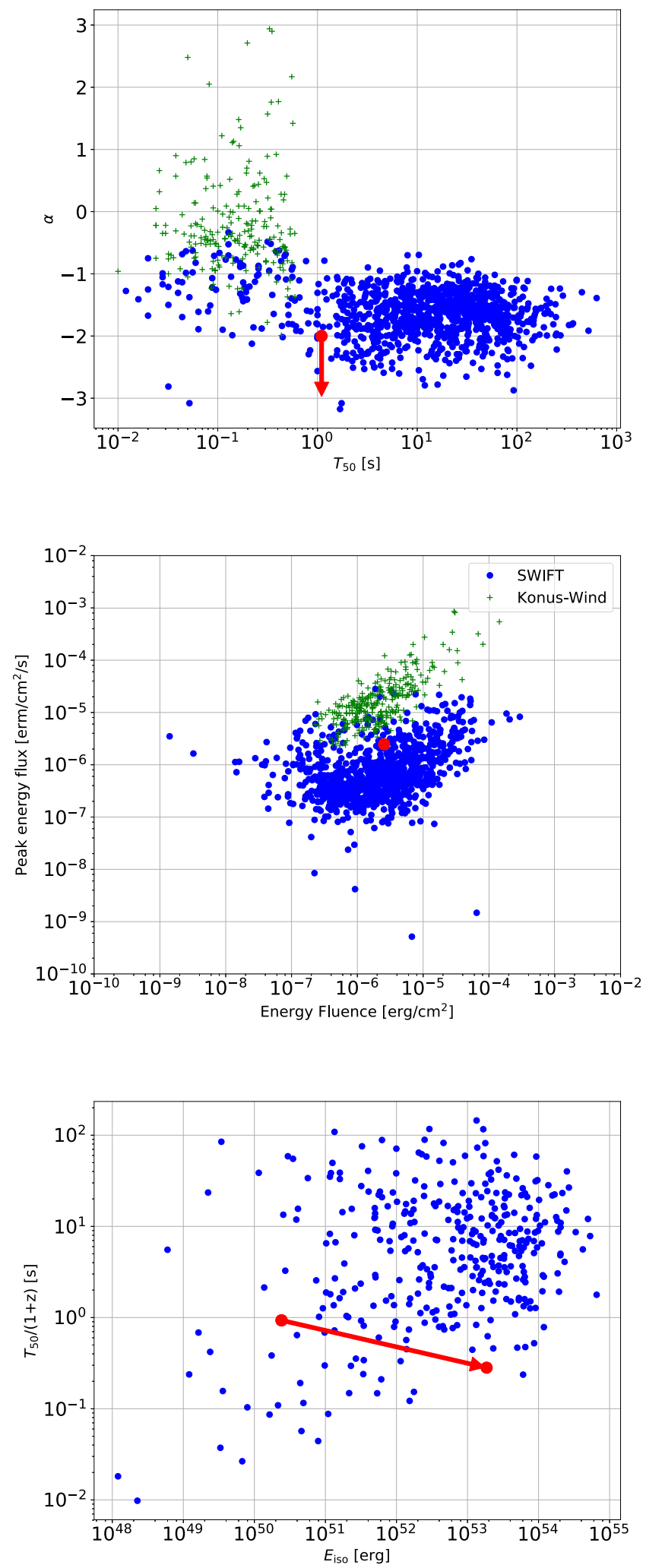

Figure 6. Panel A (top): $\alpha$ vs. $T_{50}$ for Konus-Wind (short GRBs only) and SWIFT GRBs, with approximate position of GRB 170105A. An upper limit only is possible, since there is no flux in the highest energy band and not all data are available yet. Panel B (middle) : peak energy vs. energy fluence for Konus-Wind (short GRBs only), SWIFT, and GRB 170105A. Panel C (bottom) : $E_{\text {iso }}$ vs. $T_{50} /(1+z)$ for SWIFT and GRB 170105A, where $z$ has been chosen to span from $z=0.18$ (median redshift for GW 170104) to $z=2.9$ (the arrow points to higher redshift). 
day away from the GW 170104, hence the Poisson probability of 1 or more GRBs per one-day period is $p=1-e^{-r_{1}}=0.53$ (where $\left.r_{1}=0.75\right)$. The LIGO sky map has an area of 2000 square degrees (90\% enclosed probability, from the LALInference map; Veitch et al. 2015) or about 0.05 of the sky. Therefore the rate of GRBs within one day and 2000 square degrees provides a probability of coincidence of (for $r_{2}=0.05$ ) $p=1-e^{-r_{1} r_{2}}=0.04$. This is the probability in $3 \mathrm{D}$ - the $2 \mathrm{D}$ location on the sky and in the temporal window of one day. In other words, a chance coincidence is unlikely at $2.1 \sigma$ level, but not ruled out.

However, it is worth considering that if GRB 170105A were associated with ATLAS17aeu, then there are other properties that reduce the chance of coincidence. The rate of Swift GRBs that have $m<17$ optical afterglows is $30 \%$ of all Swift GRBs from Cenko et al. (2009). We chose this value of $m<17$ since Cenko et al. (2009) defined their afterglow distribution at $t=1000 \mathrm{~s}$ after the GRB, and when we extrapolate ATLAS17aeu back to $t=1000 \mathrm{~s}$, it comfortably sits above this flux level. With only $30 \%$ of GRBs having bright afterglows, this reduces the probability of an optically bright GRB within $24 \mathrm{hr}$ within the sky annulus to $p=1-e^{-r_{1} r_{2} r_{3}}=0.01$ (with $r_{3}=0.3$ ) In other words, a chance coincidence is unlikely at the $2.6 \sigma$ level. One could also propose that the host galaxy of ATLAS17aeu (Galaxy A) is unusually faint for a GRB host, in that Savaglio et al. (2009) find that only $13 \%$ of GRBs are found in galaxies fainter than $r=25$. Hence if we fold this into the Poisson probability equation, we reduce the expectation value by a factor of $r_{4}=0.13$, giving $p=1-e^{-r_{1} r_{2} r_{3} r_{4}}=0.001$. This would imply that a chance coincidence is unlikely at the $99.9 \%$ level, or $3.3 \sigma$. However, we recognize that inclusion of every property of GRB 170105A simply serves to reduce the probability and therefore reduces the meaning and viability of the calculation. We therefore simply state that the chance of a long GRB within the time and 2D sky-area window for GW 170104 is $p=0.04$. While we have a 4D location (in space and time) for GW 170104, we lack a definitive distance to GRB 170105A. Such a measurement would provide a more definitive answer.

While the probability of a chance coincidence of the GW even with GRB 170105A (which has ATLAS17aeu as its afterglow) is low, there are further reasons to be cautious about drawing a conclusion of a causal link. We lack a quantitative physical mechanisms to produce a GRB and multiwavelength afterglow beginning $24 \mathrm{hr}$ after the merger of two BHs of masses $31 M_{\odot}$ and $19 M_{\odot}$. Perna et al. (2016) and de Mink \& King (2017) have proposed that binary BHs could well have a circumbinary disk that still exists at the time of merger and that perturbation of this disk by the GW energy could result in accretion onto the newly formed single $\mathrm{BH}$ with energy from the mid-energy X-ray range to the mid-infrared.

To consider the plausibility that emission of the sort proposed by Perna et al. (2016) and de Mink \& King (2017) has occurred, we speculate for illustrative purposes that ATLAS17aeu is within the distance range implied by LIGO $\left(z=0.18_{-0.07}^{+0.08}\right.$ or $\left.D_{\mathrm{L}}=880_{-360}^{+460} \mathrm{Mpc}\right)$. We consider the X-ray and optical to be linked through emission from hot thermal radiation from a circumbinary disk. The measured contemporaneous fluxes at +3 days are $\nu f_{\nu}=3.7 \times 10^{-14} \mathrm{erg} \mathrm{cm}^{-2} \mathrm{~s}^{-1}$ in the optical $\left(i_{\mathrm{P} 1}=21.5\right)$ and $\nu f_{\nu}=2.9 \times 10^{-13} \mathrm{erg} \mathrm{cm}^{-2} \mathrm{~s}^{-1}$ in the X-ray. This gives a ratio of $\nu f_{\nu}^{\mathrm{X}-\text { ray }} / \nu f_{\nu}^{\text {optical }}=7.8$. The Swift-detected $\mathrm{X}$-rays were soft, as shown in Figure 3, and they peaked at $1 \mathrm{keV}$ or below. When we assume that they peak at $300 \mathrm{eV}$, we can approximately fit a thermal blackbody SED at $T_{\mathrm{BB}} \sim 200,000 \mathrm{~K}$
Table 3

Luminosities and Energies at Different Wavelengths

\begin{tabular}{llccc}
\hline \hline Wavelength & Epoch & $\nu f_{\nu}$ & $\nu L_{\nu}$ & $E_{\text {iso }}$ \\
\hline & & $\operatorname{erg~s}^{-1} \mathrm{~cm}^{-2}$ & $\operatorname{erg~s}^{-1}$ & erg \\
\hline Gamma-Rays & 57758.22 & $\ldots$ & $\ldots$ & $2.4 \times 10^{50}$ \\
Optical & 57758.39 & $1.2 \times 10^{-12}$ & $1.0 \times 10^{44}$ & $\ldots$ \\
X-rays & 57760.03 & $2.9 \times 10^{-13}$ & $2.7 \times 10^{43}$ & $\ldots$ \\
Optical & 57760.03 & $3.7 \times 10^{-14}$ & $3.4 \times 10^{42}$ & $\ldots$ \\
Radio & 57760.17 & $5.0 \times 10^{-16}$ & $4.7 \times 10^{40}$ & $\ldots$ \\
\hline
\end{tabular}

Note. The fluxes are from the sources reported in the text, and the $\nu L_{\nu}$ assumes, for illustrative purposes, the luminosity distance of GW 170104, $D_{\mathrm{L}}=880$ $\mathrm{Mpc}$. The gamma rays are measured in the band $20 \mathrm{keV}-10 \mathrm{MeV}$ and the $\mathrm{X}$-rays in the Swift band $0.3-10 \mathrm{keV}$.

(20 eV), which would peak in the EUV around $140 \AA$ A. However, a thermal blackbody spectrum is a poor fit to the Gemini afterglow spectrum and to the inferred SED from the combined ATLAS and PS1 colors and the DCT photometry of Cenko \& Troja (2017), and it does not explain the radio flux either. Therefore we can conclude that the spectral energy distribution at the time of the first (and brightest) X-ray point appears to be nonthermal, regardless of the distance of the GRB. If indeed ATLAS17aeu were within the LIGO distance range, then it has luminosities in the X-ray, optical, and radio $\left(\nu L_{\nu}\right.$ in Table 3$)$ of about $\sim 10^{42}-10^{43} \mathrm{erg} \mathrm{s}^{-1}$. This is broadly similar to the energies predicted by de Mink \& King (2017) for the circumbinary disk accretion model. The uncertainties are large and the luminosity is a strong power of $v$, the greater of the Keplerian disk velocity and the post-merger kick velocity (which is unknown), and dependent on an unknown efficiency scaling factor and the relic disk mass (see their Equation (6)). In addition, the de MinkKing model predicts that the emitting region should have a characteristic emitting temperature between $10^{6} \mathrm{~K} \lesssim T \lesssim$ $10^{7} \mathrm{~K}$ and the peak of the EM radiation should be in the medium $\mathrm{X}$-ray energy regime. This is quite compatible with the emitted energies of ATLAS17aeu (Table 3), and the delay time of several hours between the GW and energy emission is also in the broad region proposed in de Mink \& King (2017). Of course, this relies on the assumption that ATLAS17aeu is within the LIGO distance range. It is not clear if the de Mink-King model can also produce a GRB through disk accretion with a delay of $\sim 24 \mathrm{hr}$ after BH merger. It is possible that the GRB emission is a distraction, a chance coincidence, and not related to ATLAS17aeu. As shown above, this is statistically unlikely, but still plausible. A key requirement is future constraints on the redshift of the host galaxy of ATLAS17aeu, either through very deep and long integration spectra, or through a photometric redshift technique. If we were able to determine the redshift and luminosity distance of either Galaxy A or Galaxy B, and if it were to fall within the distance range of GW 170104, this would reduce the rate of such a coincidence to levels that would force consideration of a physical link. Correspondingly, if the redshift were clearly outside the GW 170104 range, a chance coincidence could be securely concluded.

Finally, we comment on how frequently ATLAS should see optical afterglows of GRBs during its normal operations. Since ATLAS typically observes five times over a 5000 square degree footprint every night, there is a quantifiable probability of the system catching a GRB afterglow. The number $N_{\mathrm{GRB}}$ 
afterglows that ATLAS is likely to detect per year is

$$
N_{\mathrm{GRB}}=3.2 \frac{R_{\mathrm{GRB}}}{365} \frac{A}{5000} \frac{w}{5} \frac{f_{\mathrm{obs}}}{0.5} \frac{C}{0.7} \mathrm{yr}^{-1},
$$

where $R_{\mathrm{GRB}}$ is the annual rate of GRBs, $A$ is the area in square degrees covered per night, $w$ is the time window in hours that GRB afterglows are typically visible above the ATLAS limit of $c, o \simeq 18.5, f_{\mathrm{obs}}$ is the fraction of GRB afterglows that are detectable by ATLAS during this period of $w$ hours, and $C$ is the fraction of clear, useful weather time that ATLAS observes. For $R_{\mathrm{GRB}}=275$, then $N=2.4$ over one year, and the major uncertainty is $f_{\text {obs }}$. Since ATLAS has been working in survey mode for approximately one year (2016 March-2017 April) and we have been processing the difference imaging routinely during this period, finding one candidate for a GRB-like afterglow (ATLAS17aeu) is not unexpected ( $p=30 \%$ of finding 1 or 0 for an expected rate of 2.4).

\section{Summary}

We have reported the discovery of the transient ATLAS17aeu that lies spatially within the GW 170104 sky map and has a first optical detection $23.2 \mathrm{hr}$ after the binary $\mathrm{BH}$ merger. Analysis of multiwavelength data from X-ray through radio indicates that it is likely to be the optical, X-ray, and radio afterglow of the gamma-ray burst GRB 170105 . The distance inferred by LIGO to GW 170104 of $D_{\mathrm{L}}=880_{-360}^{+460} \mathrm{Mpc}\left(z=0.18_{-0.07}^{+0.08}\right)$ leaves us with two possibilities.

One is that GRB 170105A and its afterglow ATLAS17aeu are simply part of the known GRB population at higher redshift than GW 170104 and a chance coincidence in time and 2D sky area. This hypothesis would not violate any constraints from the observational data, but is not uniquely proven yet. The other is that ATLAS17aeu and its associated GRB is an unusual lower redshift transient and is physically associated with GW17014. Specifically, we find the following:

1. We have detected a host galaxy, or host galaxy system, but do not have a secure redshift. For the radio flux to be consistent with a relativistic outflow and a standard GRB, the source should be $z \gtrsim 1$ (to produce a Lorentz factor $\Gamma \geqslant 5)$. The lack of Ly $\alpha$ forest absorption in the afterglow spectrum indicates a fairly secure upper redshift limit of $z \lesssim 2.9$. The GRB is soft, with extended flux in the softest Konus-Wind band of $18-70 \mathrm{keV}$ lasting about $20 \mathrm{~s}$. Therefore a soft GRB lying at $1 \lesssim z \lesssim 2.9$ is not inconsistent with any observational data, including the faint host.

2. We computed the probability that ATLAS17aeu was indeed such a chance coincidence with GW 170104, assuming it was a GRB-like event, and find this to be small (formally 2.1-3.3 $\sigma$ ). One could argue that the probability calculation is somewhat selective in its choices of what to choose as sky rates, and the most robust probability to quote for coincidence is $p=0.04$, or a chance coincidence that is significant at $2.1 \sigma$.

3. While this is low, it is not significant enough to link the two events. If a redshift and distance to the host galaxy could be established and if it were to be consistent with the LIGO range for GW 170104, then the GRB and afterglow properties would not be those of the normal
GRB population. The emitted energies in the optical, $\mathrm{X}$-ray and radio would be similar to those predicted by de Mink \& King (2017) for plausible EM signatures from coalescing BHs with a relic circumbinary disk. It is not clear if the gamma rays or significant time-delay as observed can be produced in this model.

4. Hence it is essential that a distance estimate (either spectroscopic or photometric) is measured for the faint host galaxy (at $r=25.6$ ) and its nearby companion. A redshift lying outside the LIGO range for GW 170104 would securely rule this out as a viable counterpart.

In conclusion, given the multiwavelength data combined with the rate and probability calculations, it appears that GRB 170105A and ATLAS17aeu are linked, but their energetics are most comfortably explained by a high-redshift GRB and afterglow, which is just a chance coincidence with the position and time of GW 170104. The probability of such a coincidence is low, but not uncomfortably so.

However, hypothesizing a link between this energetic electromagnetic event and GW 170104 is quite testable. If a soft gamma-ray to radio transient of something resembling ATLAS17aeu is generally produced by merging BHs (in the de Mink-King scenario), then we have had three events for which such optical coverage has been attempted (GW 150914, GW151226, and GW 170104). For the first, issues with the sky map release and observability of much of the high-probability region hindered observing. We finally covered only $4 \%$ of the region containining the source (Smartt et al. 2016b), iPTF covered about 2.5\% (Kasliwal et al. 2016), and the Dark Energy Camera project covered 3\% (Soares-Santos et al. 2016), hence we can discount this as a quantitative search. For the latter two, we covered $31 \%$ and $43 \%$ of the respective regions with Pan-STARRS and ATLAS (see Smartt et al. 2016a). The Dark Energy Camera and J-GEM teams covered 3\% and 29\% of the GW151226 map (Cowperthwaite et al. 2016; Yoshida et al. 2017, respectively) and there was substantial overlap between our Pan-STARRS/ATLAS coverage and that of J-GEM. Assuming that for any well-observed event we have a $40 \%$ coverage of the sky map, then using the binomial distribution (probability of $d$ detections in $n$ trial events), the probability of obtaining two or more detections when we observe 8 LIGO events is 93\% (approximately $2 \sigma$ ). The corollary is that to rule out optical electromagnetic counterparts that accompany binary $\mathrm{BH}$ mergers at the $3 \sigma$ confidence level requires at least 11 events with $40 \%$ sky probability coverage. It behooves the EM community to either increase the sky survey coverage (for example, reaching $75 \%$ probability coverage would then require only 4 events), or for LIGO and Virgo, and potentially KAGRA, to shrink the size of the sky maps by factors of around $\sim 2$. It is also essential that accurate maps are released promptly (within $1 \mathrm{hr}$ of event detection) given the timescales for decline for ATLAS17aeu and the practical limitations of optical observations (e.g., daylight hours and weather).

With thanks to Dimitry Svinkin for insights into the publicly available gamma-ray data. PS1 and ATLAS are supported by the following NASA Grants: NNX08AR22G, NNX12AR65G, NNX14AM74G, and NNX12AR55G. The Pan-STARRSLIGO effort is in collaboration with the LIGO Consortium and supported by the University of Hawaii and Queen's University Belfast. The Pan-STARRS1 Sky Surveys have been 
made possible through contributions by the Institute for Astronomy, the University of Hawaii, the Pan-STARRS Project Office, the Max Planck Society and its participating institutes, the Max Planck Institute for Astronomy, Heidelberg and the Max Planck Institute for Extraterrestrial Physics, Garching, The Johns Hopkins University, Durham University, the University of Edinburgh, the Queen's University Belfast, the Harvard-Smithsonian Center for Astrophysics, the Las Cumbres Observatory Global Telescope Network Incorporated, the National Central University of Taiwan, the Space Telescope Science Institute, and the National Aeronautics and Space Administration under Grant No. NNX08AR22G issued through the Planetary Science Division of the NASA Science Mission Directorate, the National Science Foundation Grant No. AST-1238877, the University of Maryland, Eotvos Lorand University (ELTE), and the Los Alamos National Laboratory. S.J.S. acknowledges funding from the European Research Council under the European Union's Seventh Framework Programme (FP7/2007-2013)/ERC Grant agreement $\mathrm{n}^{\mathrm{o}}$ [291222] and STFC grant ST/P000312/1. T.W.C. acknowledges the support through the Sofia Kovalevskaja Award to P. Schady from the Alexander von Humboldt Foundation of Germany, and thanks to T. Krühler, Y.-C. Pan, and J. Graham for helping with the GMOS data reduction. M.W.C. is supported by National Science Foundation Graduate Research Fellowship Program under NSF grant number DGE 1144152. K.M. acknowledges support from the STFC through an Ernest Rutherford Fellowship. Based partly on observations obtained at the Gemini Observatory under programmes GN-2017A-Q23 and GN-2016B-Q-2. Gemini is operated by the Association of Universities for Research in Astronomy, Inc., under a cooperative agreement with the NSF on behalf of the Gemini partnership: the National Science Foundation (United States), the National Research Council (Canada), CONICYT (Chile), Ministerio de Ciencia, Tecnología e Innovación Productiva (Argentina), and Ministério da Ciência, Tecnologia e Inovação (Brazil). Some of the data presented herein were obtained at the W.M. Keck Observatory, which is operated as a scientific partnership among the California Institute of Technology, the University of California and the National Aeronautics and Space Administration. The Observatory was made possible by the generous financial support of the W.M. Keck Foundation.

Facilities: ATLAS, PS1, Gemini-GMOS, Keck-DEIMOS.

Software: DOPHOT (Schechter et al. 1993), TPHOT, IRAF (Tody 1993), WebPIMMS/HEASARC.

\section{ORCID iDs}

B. Stalder (iD https:// orcid.org/0000-0003-0973-4900 J. Tonry (iD https://orcid.org/0000-0003-2858-9657

S. J. Smartt (1) https://orcid.org/0000-0002-8229-1731

M. Coughlin (iD https://orcid.org/0000-0002-8262-2924

K. C. Chambers (i) https://orcid.org/0000-0001-6965-7789

C. W. Stubbs (D) https://orcid.org/0000-0003-0347-1724

T.-W. Chen (i) https://orcid.org/0000-0003-1532-0149

D. R. Young (i) https://orcid.org/0000-0002-1229-2499

M. E. Huber (iD https://orcid.org/0000-0003-1059-9603

H. Flewelling (D) https://orcid.org/0000-0002-1050-4056
E. A. Magnier (i) https://orcid.org/0000-0002-7965-2815

C. Waters (iD https://orcid.org/0000-0003-1989-4879

R. Wainscoat (iD https://orcid.org/0000-0002-1341-0952

D. Sanders (1D https://orcid.org/0000-0002-1233-9998

C. Inserra (1) https://orcid.org/0000-0002-3968-4409

K. Maguire (ib https://orcid.org/0000-0002-9770-3508

R. Kotak (iD https://orcid.org/0000-0001-5455-3653

\section{References}

Abbott, B. P., Abbott, R., Abbott, T. D., et al. 2016a, ApJL, 826, L13 Abbott, B. P., Abbott, R., Abbott, T. D., et al. 2016b, PhRvL, 116, 061102 Abbott, B. P., Abbott, R., Abbott, T. D., et al. 2017, PhRvL, 118, 221101 Anderson, G. E., van der Horst, A. J., Staley, T. D., et al. 2014, MNRAS, 440, 2059

Bailey, J. 1975, JBAA, 86, 30

Berger, E. 2014, ARA\&A, 52, 43

Berger, E., Leibler, C. N., Chornock, R., et al. 2013, ApJ, 779, 18

Bhalerao, V., Kasliwal, M. M., Bhattacharya, D., et al. 2017, ApJ, 845, 152

Cenko, S., \& Troja, E. 2017, GCN, 20397, 1

Cenko, S. B., Kelemen, J., Harrison, F. A., et al. 2009, ApJ, 693, 1484

Chambers, K. C., Magnier, E. A., Metcalfe, N., et al. 2016, arXiv:1612.05560

Connaughton, V., Burns, E., Goldstein, A., et al. 2016, ApJL, 826, L6

Corsi, A., Kasliwal, M., Frail, D., \& Palliyaguru, N. 2017, GCN, 20396, 1

Cowperthwaite, P. S., Berger, E., Soares-Santos, M., et al. 2016, ApJL, 826, L29

de Mink, S. E., \& King, A. 2017, ApJL, 839, L7

Evans, P. A., Beardmore, A. P., Page, K. L., et al. 2009, MNRAS, 397, 1177

Evans, P. E. K., Barthelmy, S., Beardmore, A., \& Breeveld, A. 2017a, GCN, 20415, 1

Evans, P. E. K., Barthelmy, S., Beardmore, A., \& Breeveld, A. 2017b, GCN, 20390, 1

Fynbo, J. P. U., Jakobsson, P., Prochaska, J. X., et al. 2009, ApJS, 185, 526 Galama, T. J., Briggs, M. S., Wijers, R. A. M. J., et al. 1999, Nat., 398, 394 Greiner, J., Burgess, J. M., Savchenko, V., \& Yu, H.-F. 2016, ApJL, 827, L38 Hook, I. M., Jørgensen, I., Allington-Smith, J. R., et al. 2004, PASP, 116, 425 Kasliwal, M., Adams, S., Vedantham, H., et al. 2017, GCN, 20393, 1

Kasliwal, M. M., Cenko, S. B., Singer, L. P., et al. 2016, ApJL, 824, L24

Krühler, T., Malesani, D., Fynbo, J. P. U., et al. 2015, A\&A, 581, A125

Marcinkowski, R., Xiao, H., \& Hajdas, W. 2017, GCN, 20387, 1

Melandri, A., Piranomonte, S., Branchesi, M., et al. 2017a, GCN, 20735, 1

Melandri, A., Piranomonte, S., D'Avanzo, P., et al. 2017b, GCN, 20416, 1

Mooley, K., Fender, R., \& Horesh, A. 2017, GCN, 20425, 1

Perna, R., Lazzati, D., \& Giacomazzo, B. 2016, ApJL, 821, L18

Savaglio, S., Glazebrook, K., \& Le Borgne, D. 2009, ApJ, 691, 182

Schechter, P. L., Mateo, M., \& Saha, A. 1993, PASP, 105, 1342

Sharma, V., Bhalerao, V., Bhattacharya, D., Rao, A. R., \& Vadawale, S. 2017, GCN, 20389, 1

Singer, L. P., Chen, H.-Y., Holz, D. E., et al. 2016, ApJL, 829, L15

Smartt, S. J., Chambers, K. C., Smith, K. W., et al. 2016a, ApJL, 827, L40

Smartt, S. J., Chambers, K. C., Smith, K. W., et al. 2016b, MNRAS, 462, 4094

Smartt, S. J., Valenti, S., Fraser, M., et al. 2015, A\&A, 579, A40

Soares-Santos, M., Kessler, R., Berger, E., et al. 2016, ApJL, 823, L33

Steeghs, D., Pollacco, D., Ulaczyk, K., et al. 2017, GCN, 20434, 1

Svinkin, D., Golenetskii, S., Aptekar, R., et al. 2017, GCN, 20406, 1

Svinkin, D. S., Frederiks, D. D., Aptekar, R. L., et al. 2016, ApJS, 224, 10

Tody, D. 1993, in ASP Conf. Ser. 52, Astronomical Data Analysis Software and Systems II, ed. R. J. Hanisch, R. J. V. Brissenden, \& J. Barnes (San Francisco, CA: ASP), 173

Tonry, J., Denneau, L., Heinze, A., et al. 2017, GCN, 20382, 1

Tonry, J., Denneau, L., Stalder, B., et al. 2016, ATel, 8680, 1

Tonry, J. L. 2011, PASP, 123, 58

Veitch, J., Raymond, V., Farr, B., et al. 2015, PhRvD, 91, 042003

von Kienlin, A., Meegan, C. A., Paciesas, W. S., et al. 2014, ApJS, 211, 13

Wright, D. E., Smartt, S. J., Smith, K. W., et al. 2015, MNRAS, 449, 451

Yoshida, M., Utsumi, Y., Tominaga, N., et al. 2017, PASJ, 69, 9 